\title{
Periphere arterielle Verschlusskrankheit: Epidemiologie, Komorbidität und Prognose
}

\author{
Holger Lawall, Curt Diehm, Ulrich Hoffmann, Holger Reinecke
}

\section{Was ist neu?}

- Definition, Prävalenz und Inzidenz: Die Prävalenz der PAVK nimmt in Deutschland und weltweit zu. Mit höherem Lebensalter (> 70 Lj.) steigt sie geschlechtsunabhängig auf etwa $20 \%$.

- Diagnostik: Ist die Messung des Knöchel-Arm-Index (ABI) nicht aussagekräftig, ermöglicht die Messung der Zehendruckwerte mit Bestimmung des Zehen-Arm-Index (TBI) eine zuverlässige Aussage zur Hämodynamik. $\mathrm{ABI}$ und TBI dienen dabei nicht nur zur Diagnosestellung einer PAVK, sondern stehen in linearer Korrelation zur kardiovaskulären Morbidität und Letalität.

- Komorbiditäten: Eine begleitende Herzinsuffizienz verschlechtert die Offenheitsraten nach primär erfolgreicher Revaskularisation und die Gesamtprognose von Patienten mit PAVK. Neben einem Diabetes mellitus sind fortgeschrittene chronische Nierenerkrankungen hinsichtlich der Progression und Prognose der PAVK von herausragender Bedeutung.

- Verlauf und Prognose: Bei stationär behandelten Patienten nimmt der Anteil der Patienten mit kritischer Extremitätenischämie in den letzten 10 Jahren kontinuierlich zu, ebenso die Anzahl von endovaskulären Eingriffen. Unverändert hoch ist allerdings die Mortalitätsrate hospitalisierter Patienten mit kritischer Ischämie.

- Unterbehandlung und mangelndes Wissen über die Erkrankung: Im Vergleich zur KHK sind Patienten mit PAVK unterbehandelt. Das Bewusstsein und die Kenntnisse über die Erkrankung sind bei den Betroffenen weiterhin gering. globale Prävalenz der PAVK (Knöchel-Arm-Index $[A B I] \leq 0,9)$ wurde 2010 aus Daten eines systematischen Reviews mit mathematischen Schätzmethoden auf 202 Millionen Menschen geschätzt.

Zwischen 2000 und 2010 hat sich die Inzidenz in Ländern mit niedrigem und mittlerem Einkommen um 28,7\% erhöht, in Ländern mit hohem Einkommen um 13,1\% [4]. In Deutschland stieg die Zahl der stationären Behandlungsfälle mit PAVK, wie eine aktuelle Untersuchung anhand von DRGAufnahmediagnosen nachweisen konnte: Während 2005 noch 2,7\% aller Hospitalisierungen auf eine PAVK zurückzuführen waren, stieg dieser Anteil 2009 auf 3,0\%. Der Anstieg war mit 32\% besonders ausgeprägt im Stadium IV zu beobachten [5].

\section{Diagnostik}

Die Prävalenz der asymptomatischen PAVK kann in der Allgemeinbevölkerung mit nicht-invasiven Untersuchungsmethoden erfasst werden. Am häufigsten wird die Messung des ABI mittels des Dopplerverschlussdrucks eingesetzt. Der Grenzwert für die Diagnose der PAVK wird bei $<0,9$ festgelegt. Die Sensitivität eines ABI-Werts $<0,9$ für das Vorliegen einer mindestens $50 \%$-igen Gefäßstenose (verifiziert mit dem Goldstandard Angiografie) beträgt nahezu 95\% in Ruhe, die Spezifität fast $100 \%$ [1]. Systematische ABI-Bestimmungen nach einem standardisierten Belastungstest finden eine um etwa 30\% höhere Prävalenz der PAVK. Eine Abnahme des ABI um 15-20\% nach Belastung im Vergleich zum Wert in Ruhe gilt dabei als beweisend für eine PAVK.

Für epidemiologische Fragestellungen wird jeweils der niedrigere der beiden systolischen Knöchelarteriendrucke (A. dorsalis pedis/A. tibialis posterior) zum Nachweis einer PAVK herangezogen. Für die Beurteilung der Hämodynamik in Ruhe und unter Belastung wird dagegen der höhere der beiden Werte benutzt. Ein erniedrigter $\mathrm{ABI}$ ist ein zuverlässiger Indikator für eine erhöhte kardiovaskuläre Morbidität und Mortalität. Außerhalb von Hochrisikogruppen (z. B. Diabetiker, Koronarpatienten u.a.) wird ein generelles ABIScreening in der Allgemeinbevölkerung jedoch nicht empfohlen, wie ein systematisches Review aufzeigt [2].

Bei Vorliegen einer Mediasklerose kann die Bewertung des $\mathrm{ABI}$ durch falsch hohe systolische 


\begin{tabular}{ll} 
Fontaine & \\
Stadium & Klinisches Bild \\
I & asymptomatisch \\
II a & Gehstrecke $>200 \mathrm{~m}$ \\
II b & Gehstrecke $<200 \mathrm{~m}$ \\
III & ischämischer Ruheschmerz \\
IV & Ulkus, Gangrän \\
\hline
\end{tabular}

Knöchelarteriendrucke problematisch sein. Ein $\mathrm{ABI}>1,3$ spricht für das Vorliegen einer Mediakalzinose und findet sich gehäuft bei Diabetikern und Patienten mit chronischen Nierenerkrankungen. Nicht nur erniedrigte, sondern auch erhöhte ABI-Werte (>1,3) sind mit einer gesteigerten Mortalitätsrate assoziiert. Gleiches gilt für die Messung des systolischen Zehenarteriendruckes und des daraus resultierenden Zehen-Arm-Indexes (TBI), der im Falle einer Mediasklerose häufig eine verlässliche hämodynamische Einschätzung ermöglicht. Zu beachten ist, daß der TBI im Mittel um 0,2 höher liegt als der ABI [3].

\section{Klinische Relevanz \\ Neben der Bestimmung des TBI lässt sich die hämodynamische Situation der Extremitäten bei Mediasklerose auch mithilfe der Vorfuß- oszillografie einschätzen. Neben der Messung der systolischen Knöchel- oder Zehenarterien- druckmessung ist sie darüberhinaus Bestand- teil der Rutherford-Klassifikation.}

\section{Komorbiditäten}

Koronare Herzkrankheit (KHK) | Die Koinzidenz von PAVK und KHK ist Ausdruck der zugrunde liegenden generalisierten Arteriosklerose. Bei symptomatischer PAVK findet sich bei $62 \%$ der Patienten eine KHK [6]. Bei KHK-Patienten läßt sich bei $36 \%$ der Betroffenen mittels ABI-Messung eine PAVK nachweisen [7].

Der Nachweis einer PAVK ist ein sensitiver, geschlechtsunabhängiger Risikoindikator für das Vorliegen einer KHK und sollte somit regelhaft Anlass zu einer kardiologischen Abklärung geben [8]. Die Koinzidenz einer PAVK mit einer KHK ist häufig und kann durch eine kardial limitierte Gehstrecke infolge Angina pectoris oder Dyspnoe leicht übersehen werden.

Der Nachweis beider Erkrankungen verschlechtert die Prognose gegenüber dem singulären Auftreten erheblich [7]. Basierend auf Daten aus dem REACH-Register lagen nach einem Jahr Verlaufs-

\section{Rutherford}

$\begin{array}{lll}\text { Grad } & \text { Kategorie } & \text { klinische Bild } \\ 0 & 0 & \text { asymptomatisch } \\ \text { I } & 1 & \begin{array}{l}\text { leichte Claudicatio intermittens } \\ \text { I }\end{array} \\ \text { I } & 3 & \text { mäßige Claudicatio intermittens } \\ \text { II } & 4 & \text { schwere Claudicatio intermittens } \\ \text { III } & 5 & \text { ischämischer Ruheschmerz } \\ \text { III } & 6 & \text { kleinflächige Nekrose } \\ \text { großlächige Nekrose }\end{array}$

Tab. 1 Klassifikation der

beobachtung tödliche und nichttödliche kardiovaskuläre Ereignisse bei alleiniger KHK bei 13 und stiegen auf $23 \%$ bei koinzidenter KHK und PAVK.

PAVK nach den FontaineStadien und RutherfordKategorien.

Herzinsuffizienz | In einer Substudie der CORONAStudie an Patienten mit systolischer Herzinsuffizienz zeigten in der multivariaten Analyse Patienten mit intermittierender Claudicatio sowohl ein erhöhtes Mortalitätsrisiko als auch ein erhöhtes Risiko für tödliche und nicht-tödliche Myokardinfarkte im Vergleich zu Patienten ohne begleitende PAVK. Eine große Meta-Analyse bei Koronarpatienten mit ischämischer Kardiomyopathie und reduzierter linksventrikulärer Auswurffraktion unterstreicht die schlechte Prognose bei Vorhandensein einer PAVK [9].

Eine Herzinsuffizienz zusätzlich zur PAVK verschlechtert die periphere Durchblutung durch das verminderte Herzzeitvolumen und wirkt sich negativ auf den Extremitätenerhalt aus. Nach endovaskulärer Intervention zeigen Patienten mit begleitender systolischer Herzinsuffizienz (Ejektionsfraktion $[E F]<40 \%$ ) schlechtere primäre 1-Jahres-Offenheitsraten als Patienten mit einer $\mathrm{EF}>40 \%$ (43,2 vs. 56,6\%). Vergleichbare Unterschiede ergaben sich für die sekundären Offenheitsraten [10].

Patienten mit PAVK und Herzinsuffizienz sind insgesamt körperlich schlechter belastbar und im geringeren Maße durch ein körperliches Training verbesserungsfähig. Dies wies eine Untersuchung innerhalb der HF-ACTION-Studie an Patienten mit Herzinsuffizienz NYHA II-IV und einer EF $\leq 35 \%$ nach. Patienten mit begleitender PAVK zeigten geringere Verbesserungen der körperlichen Leistungsfähigkeit durch ein strukturiertes körperliches Trainingsprogramm als Patienten ohne begleitende PAVK. Die PAVK stellte zudem einen unabhängigen Prädiktor für Mortalität oder Hospitalisierung dar(Hazard ratio 1,31,95\%-Konfidenzintervall 1,06-1,62) [11].

Diabetes mellitus | Ein Diabetes mellitus ist unabhängig vom Diabetestyp mit einem erhöhten Risiko für arteriosklerotische Gefäßerkrankungen verbunden und verdoppelt nahezu die Wahr- 
Abb. 1 Ergebnisse der getABI-Studie: Mortalitätsraten nach 1,3 , und 5 Jahren bei symptomatischen und asymptomatischen PAVK-Patienten im Vergleich zu Patienten ohne PAVK [20]. scheinlichkeit für eine PAVK [5]. Diabetiker entwickeln dabei vor allem eine distal ausgeprägte PAVK. Die Notwendigkeit einer koronaren bzw. peripheren Bypass-OP ist im Vergleich zu NichtDiabetikern erhöht [12]. Die Rate an kardiovaskulären Ereignissen erhöht sich bei Patienten mit Typ-2-Diabetes mit steigenden $\mathrm{HbA}_{1 \mathrm{c}}$. In einem systematischen Review [13] betrug das gepoolte relative Risiko pro Anstieg des $\mathrm{HbA}_{1 \mathrm{c}}$ um $1 \%$ für

- die Gesamtmortalität 1,15 (1,11-1,20),

- eine kardiovaskuläre Erkrankung ebenfalls 1,15 (1,10-1,20),

- eine Herzinsuffizienz $1,11(1,06-1,17)$ und

- eine PAVK 1,29 (1,18-1,40).

Eine schlechte Stoffwechsellage ist mit höheren Reverschlussraten und schlechteren klinischen Ergebnissen nach infrapoplitealer endovaskulärer Therapie assoziert [14].

Eine dänische Fall-Kontroll-Studie konnte keinen Nutzen einer intensivierten Diabetes-Therapie gegenüber einer konventionellen Therapie im Hinblick auf das Neuauftreten einer PAVK nach 6 Jahren feststellen [15]. Demgegenüber ergaben Langzeituntersuchungen der Steno- und DCCTStudie sowie eine große Meta-Analyse aus Interventionsstudien zur intensivierten Therapie des Typ-2-Diabetes Hinweise für einen Nutzen einer intensivierten Diabetestherapie hinsichtlich der Reduktion von Major-Amputationen bei Diabetikern [16].

Die Nationale Versorgungsleitlinie Diabetes fordert im Diagnosealgorithmus bei klinischem Verdacht auf eine PAVK aufgrund des engen Zusammenhangs von PAVK und Diabetes ein Screening mittels Bestimmung des ABI. Gemäß den Praxisleitlinienempfehlungen der Deutschen Diabetesgesellschaft sollten alle Patienten mit Diabetes mellitus und dem Risiko für die Entstehung eines diabetischen Fußsyndroms neben der klinischen Fußuntersuchung auch eine Messung der systolischen Knöcheldrücke erhalten. Bei eingeschriebenen Patienten mit Diabetes mellitus im Disease-Manage-

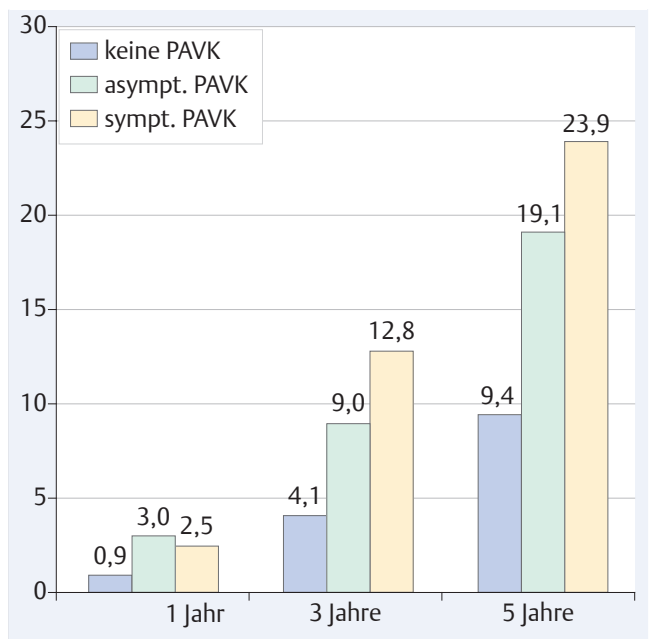

ment-Programm (DMP) ist eine jährliche $\mathrm{ABI}-$ Messung vorgesehen.

Chronische Nierenerkrankungen | Mit abnehmender glomerulärer Filtrationsrate (GFR) steigt die kardiovaskuläre Morbidität und Mortalität. Eine chronische Nierenerkrankung ist neben den „klassischen” Risikofaktoren ein unabhängiger, „dosis-abhängiger” Risikofaktor für kardiovaskuläre Ereignisse und Tod [17]. Die Prävalenz der PAVK steigt mit abnehmender Nierenfunktion: Während im Stadium 3 der Niereninsuffizienz bei bis zu 15\% der Patienten eine PAVK gefunden wird, beträgt dieser Anteil bei dialysepflichtigen Patienten nahezu 50\% [18].

Aufgrund der häufig diffusen und peripher gelegenen arteriellen Obstruktionen sind die Ergebnisse der endovaskulären und offen chirurgischen Revaskularisation bei niereninsuffizienten Patienten mit fortschreitender Nierenerkrankung signifikant schlechter als bei Nierengesunden. Eine Nierenerkrankung senkt dabei die Wahrscheinlichkeit eines amputationsfreien Überlebens bei Patienten mit kritischer Extremitätenischämie drastisch über einen Beobachtungszeitraum von 3 Jahren (HR 3,68 [1,51-8,94]) [19].

\section{Klinische Relevanz}

- Patienten mit PAVK sollten hinsichtlich einer KHK oder weiterer arteriosklerotischer Gefäßerkrankungen in anderen vaskulären Territorien abgeklärt werden.

- Eine Herzinsuffizienz verschlechtert die Prognose der Gefäßoffenheit sowie die Gesamtprognose.

- Diabetes mellitus ist nach dem Rauchen der wesentliche Risikofaktor für die Manifestation und Progression der PAVK. Diabetiker sollten auf PAVK gescreent werden.

- Chronische Nierenerkrankungen sind ein unabhängiger Risikofaktor für PAVK. Sie verschlechtern die Prognose hinsichtlich des amputationsfreien Überlebens erheblich.

\section{Verlauf und Prognose}

Asymptomatische PAVK | Sowohl ein pathologisch erniedrigter als auch der pathologisch erhöhter $A B I$ ist ein unabhängiger Indikator für kardiovaskuläre Morbidität und Mortalität. Dies gilt auch für klinisch asymptomatische Patienten, wie die Daten der getABI-Studie zeigen $(\checkmark$ Abb. 1) [20].

Symptomatische PAVK | Das Schicksal der Patienten mit Claudicatio wird vor allem von kardialen und zerebralen Ereignissen bestimmt. So betra- 
gen die 4-Jahres-Ereignisraten bei Patienten mit einer Claudiatio intermittens (IC) für

- Sterblichkeit $19 \%$,

- überlebte Herzinfarkte 7\% und

- ischämische Schlaganfälle 5\%.

Das Risiko einer kritischen Extremitätenischämie ist bei diesen Patienten eher gering. Nur etwa $4 \%$ erleiden eine Amputation innerhalb der nächsten 4 Jahre [21].

Im Vergleich dazu haben Patienten mit kritischer Extremitätenischämie eine sehr hohe 1-, 5- und 10-Jahres-Mortalität. In einem französischen Register (COPART) mit 940 Patienten betrug die 1-Jahres-Mortalitätsrate bei Patienten mit stabiler IC 5,7\% im Vergleich zu 23,1 \% bei Patienten mit kritischer Extremitätenischämie. Die 1-JahresSterblichkeit betrug $28,7 \%$ bei Patienten mit Ulzerationen [22]. In der BASIL-Studie betrug das amputationsfreie Überleben für die Gesamtgruppe der Patienten (allesamt operabel) mit kritischer Extremitätenischämie nach einem Jahr 70\% und nach 3 Jahren 55\%. Die Mortalität nach einem Jahr lag bei etwa $20 \%$.

Bei Patienten mit Diabetes mellitus ist die Prognose noch deutlich schlechter. Mortalitäts- und Major-Amputationsraten sind gegenüber Nichtdiabetikern signifikant erhöht [12]. Im multinationalen REACH-Register wurde die Häufigkeit atherothrombotischer Erkrankungen und der damit assoziierten Risikofaktoren in der Praxis erfasst. Es umfasst mit 68000 Patienten aus 44 Ländern (davon 5645 aus Deutschland) das geografisch und hinsichtlich der Fallzahl größte Patienten-Register zur Atherothrombose. Bereits nach einem Jahr zeigte sich bei Patienten mit symptomatischer PAVK eine signifikante Übersterblichkeit gegenüber Patienten mit koronarer oder zerebrovaskulärer Gefäßerkrankung. Die Mortalitätsrate beträgt bei PAVK-Patienten jährlich 2,4 gegenüber 1,8\% bei KHK. Damit einher geht eine Amputationsrate von $1,3 \%$ pro Jahr.

Daten des Statistischen Bundesamtes aus DRG-Diagnosen zu Sterblichkeiten und In-Hospital-Behandlungen in Deutschland wurden von Malyar et al. ausgewertet [5]. So sank die Major-Amputationsrate in Deutschland von 4,6\% in 2005 auf 3,5\% in 2009. Parallel stieg die Rate an Minor-Amputationen minimal von 5,0\% in 2005 auf 5,1\% in 2009. Während der untersuchten Zeitspanne blieb die intrahospitale Mortalität von Patienten mit einer Claudicatio konstant $(2,2 \%)$ und die von CLI-Patienten fiel von $9,8 \%$ auf $8,4 \%$. Im Gegensatz zu diesen nur leichten, anteiligen Veränderungen stieg die absolute Anzahl, bedingt durch die deutlich zunehmende Zahl von PAVK-Patienten, z.T. erheblich: In Tab. 2 sind wesentliche Veränderungen mit Blick bei stationär behandelten Patienten in Deutschland von 2005-2009 aufgelistet [5].
PAVK Stadium / Behandlung

$\begin{array}{ll}\text { stationäre Behandlungsfälle } & +21 \% \\ \text { kritische Extremitätenischämie } & +30 \% \\ \text { endovaskuläre Therapien } & +46 \% \\ \text { Thrombembolektomien } & +69 \% \\ \text { Endarterektomien } & +42 \% \\ \text { Patchplastiken } & +21 \% \\ \text { periphere Bypassverfahren } & -2 \% \\ \text { Major-Amputationen } & -1,5 \% \\ \text { Minor-Amputationen } & +24 \%\end{array}$

Die stationäre Mortalitätsrate (30-Tage) bei kritischer Ischämie ist auch heute noch hoch und liegt zwischen 4 und $8 \%$ aller betroffenen Patienten. Erschreckenderweise hat etwa die Hälfte aller Patienten mit kritischer Ischämie vor einer MajorAmputation keine radiologische Bildgebung bekommen [23].

\section{Klinische Relevanz}

Auch Patienten mit asymptomatischer PAVK haben bereits eine deutlich erhöhte kardiovaskuläre Morbidität und Mortalität. Patienten mit kritischer Extremitätenischämie haben eine schlechte Prognose hinsichtlich Beinerhalt, Überleben und amputationsfreiem Überleben.

\section{Unterbehandlung und mangelndes Wissen über die Erkrankung}

Eine Vielzahl von Untersuchungen belegt, dass Patienten mit PAVK im Vergleich zu Patienten mit KHK hinsichtlich der medikamentösen Sekundärprophylaxe unterbehandelt sind. Daten der getABIStudie zeigten beispielsweise, dass zwei Drittel der Patienten mit KHK, aber nur die Hälfte der Patient mit PAVK, einen Thrombozytenfunktionshemmer erhielten. Bei der Therapie mit Statinen fand sich mit $46 \%$ der Patienten mit KHK vs. 23\% der Patienten mit symptomatischer PAVK eine ähnliche Situation.

In den USA wurde eine repräsentative Gruppe von 2501 Erwachsenen ab dem 50. Lebensjahr zu den Themen PAVK, Risikofaktoren für Herz-KreislaufErkrankungen und anderen kardiovaskulären Grunderkrankungen befragt. Lediglich 26\% der Patienten wussten, was eine PAVK ist. Der Hälfte dieser Gruppe war nicht bewusst, dass Diabetes mellitus und Rauchen das Risiko für eine PAVK erhöhen. Nur einer von vier Befragten wusste, dass die PAVK eine erhöhte Mortalität infolge von
Tab. 2 Veränderungen bei der Behandlung der PAVK in Deutschland von 2005-2009.

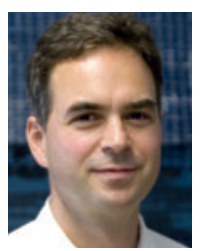

Dr. med. Holger Lawall ist Internist in der Praxis für Herzkreislauferkrankungen, Ettlingen, sowie leitender Arzt für Diabetes und Angiologie an der Max-Grundig Klinik Bühlerhöhe, Bühl. Holger.Lawall@gmail.com

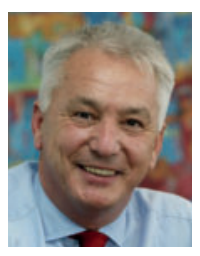

Prof. Dr. med. Curt Diehm ist Internist der Praxis für Herzkreislauferkrankungen, Ettlingen, sowie ärztlicher Direktor an der Max-Grundig Klinik Bühlerhöhe, Bühl. curt.diehm@gmail.com 


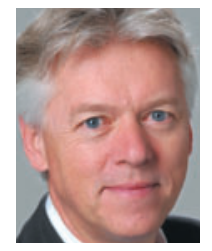

Prof. Dr. med. Ulrich Hoffmann ist Leiter der Sektion Angiologie am Gefäßzentrum der Medizinischen Klinik und Poliklinik IV, Klinikum der Ludwig-MaximiliansUniversität, München. ulrich.hoffmann@med. uni-muenchen.de

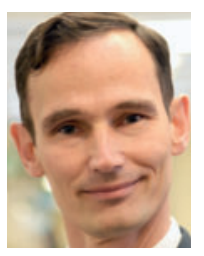

Prof. Dr. med. Holger Reinecke ist Leiter der Departments für Kardiologie und Angiologie, Universitätsklinikum Münster. holger.reinecke@ ukmuenster.de
Herzinfarkten und Schlaganfällen mit sich bringt. Nur $14 \%$ aller Befragten war klar, dass eine PAVK zur Amputation führen kann. Mangelndes Wissen bzw. fehlende Aufmerksamkeit für das Problem der PAVK zeigten insbesondere Befragte, die aufgrund ihrer Risikofaktorenkonstellation und vorhandener Grunderkrankungen das höchste Risiko für eine PAVK aufwiesen. Inwieweit die Daten auf Deutschland übertragbar sind, bleibt offen.

Die verschiedenen regionalen und überregionalen Veranstaltungskampagnen zur PAVK (z.B. „Verschlusssache PAVK“ der Deutschen Gesellschaft für Angiologie - Gesellschaft für Gefäßmedizin e.V.) weisen aber auch hier auf eine große Unkenntnis und Informationsdefizite bei Patienten und deren Angehörigen hin.

\section{Klinische Relevanz \\ Patienten mit PAVK sind hinsichtlich ihrer Risikofaktoren und Begleiterkrankungen unterbehandelt. Die Bedeutung der PAVK wird von Ärzten und Patienten unterschätzt.}

\section{Interessenkonflikt}

Die Autoren geben an, dass kein Interessenkonflikt besteht.

\section{Literatur}

1 Xu D, Zou L, Xing Y et al. Diagnostic value of ankle-brachial index in peripheral arterial disease: a meta-analysis. Can J Cardiol 2013; 29: 492-498

2 Alahdab F, Wang AT, Elraiyah TA et al. A systematic review for the screening for peripheral arterial disease in asymptomatic patients. J Vasc Surg 2015; 61: 42S-53S

3 Huyn S, Forbang NI, Allison MA et al. Ankle-brachial index, toe-brachial index, and cardiovascular mortality in persons with and without diabetes mellitus. J Vasc Surg 2014; 60: 390-395

4 Fowkes FG, Rudan D, Rudan I et al. Comparison of global estimates of prevalence and risk factors for peripheral artery disease in 2000 and 2010: a systematic review and analysis. Lancet 2013; 382: 1329-1340

5 Malyar N, Furstenberg T, Wellmann J et al. Recent trends in morbidity and in-hospital outcomes of in-patients with peripheral arterial disease: a nationwide population-based analysis. Eur Heart ] 2013; 34: 2706-2714

6 Chou SW, Kim BG, Kim DK et al. Prediction of coronary artery disease in patinets with lower extremity peripheral artery disease. Int Heart J 2015; 56: 209-212

7 Bell AD, Roussin D, Popvici-Toma D et al. The value of routine screening for peripheral arterial disease in stable outpatients with history of corornary artery and cerebrovascular disease. Int J Clin Pract 2013; 67: 996-1004

8 Subherwal S, Patel MR, Kober L et al. Peripheral arterial disease is a coronary heart disease risk equivalent among both men and women : results from a nationwide study. Eur J Prev Cardiol 2015; 22: 317-325

9 Inglis SC, Bebchuk J, Al-Suhaim Sa et al. Peripheral arterial disease and outcomes after myocardial infarction: an individual-patient meta-analysis of 28771 patients in CAPRICORN, EPHESUS, OPTIMAAL and VALIANT. Int J Cardiol 2013; 168: 1094-1101

10 Meltzer AJ, Shrikhande G, Gallagher KA et al. Heart failure is associated with reduced patency after endovascular intervention for symptomatic peripheral arterial disease. J Vasc Surg 2012; 55 : 353-362

11 Jones WS, Clare R, Ellis SJ et al. Effect of peripheral arterial disease on functional and clinical outcomes in patients with heart failure (from HF-ACTION). Am J Card 2011; 108: 380-384

12 Ryden L, Grant PJ, Anker Sd et al. ESC guidelines on diabetes, pre-diabetes, and cardiovascular diseases developed in collaboration with the EASD - summary. Diab Vasc Dis Res 2014; 11: 133-173

13 Zhang Y, Hu G, Yuan Z, Chen L. Glycosylated hemoglobin in relationship to cardiovascular outcomes and death in patients with type 2 diabetes: a systematic review and meta-analysis. PloS one 2012; 7: e42551

14 Singh S, Armstrong EJ, Sherif W et al. Association of elevated fasting glucose with lower patency and increased major adverse limb events among patients with diabtes undergoing infrapopliteal ballon angioplasty. Vasc Med 2014; 19: 307-314

15 Charles M, Ejskjaer N, Witte DR et al. Prevalence of neuropathy and peripheral arterial disease and the impact of treatment in people with screen-detected type 2 diabetes: the ADDITION-Denmark study. Diabetes Care 2011; 34: 2244-2249

16 Ray KS, Seshasai SR, Wijesuriya S et al. Effect of intensive control on cardiovascular outcomes and death in patients with diabetes mellitus: a meta-analysis of randomised controlled trials. Lancet 2009; 373: 1765-1772

17 Garamilla B, Hirsch AT. Peripheral artery disease and chronic kidney disease: clinical synergy to improve outcomes. Adv Chronic Kidney Dis 2014; 21: 460-471

18 Garimella PS, Hart PD, O’Hare A et al. Peripheral artery disease and CKD: a focus on peripheral artery disease as a critical component of CKD care. Am J Kidney Dis 2012; 60: 641-654

19 Engelhardt M, Boos J, Bruijnen $\mathrm{H}$ et al. Critical limb ischaemia: initial treatment and predictors of amputation-free survival. Eur J Vasc Endovasc Surg 2012; 43: 55-61

20 Diehm C, Allenberg JR, Haberl R et al. High all-cause mortality in patients with peripheral arterial disease in primary care: five-year results of the getABI Study. Circulation 2007; 116: II 841-b (Suppl.)

21 Golomb BA, Dang TT, Criqui MH. Peripheral arterial disease: morbidity and mortality.Circulation 2006; 114; 688-699

22 Cambou JP, Aboyans V, Constans J et al. Characteristics and outcome of patients hospitalised for lower extremity peripheral artery disease in France: the COPART Registry. Eur J Vasc Endovasc Surg 2010; 39: $577-585$

23 Reinecke H, Unrath M, Freisinger E et al. Peripheral arterial disease and critical limb ischaemia: still poor outcomes and lack of guideline adherence. Eur Heart J 2015; 36: 932-938
DOI 10.1055/s-0041-107064

Dtsch Med Wochenschr 2015; 140: 1798-1802

(c) Georg Thieme Verlag KG . Stuttgart · New York.

ISSN 0012-0472 